\title{
Expression of Truncated Neurokinin-1 Receptor in Childhood Neuroblastoma is Independent of Tumor Biology and Stage
}

\author{
ALEXANDRA POHL, ROLAND KAPPLER, JAKOB MÜHLING, \\ DIETRICH VON SCHWEINITZ and MICHAEL BERGER \\ Department of Pediatric Surgery, Dr von Hauner Children's Hospital, \\ University Hospital, Ludwig-Maximilians-University Munich, Munich, Germany
}

\begin{abstract}
Background: Neuroblastoma is an embryonal malignancy arising from the aberrant growth of neural crest progenitor cells of the sympathetic nervous system. The tachykinin receptor 1 (TACR1) - substance P complex is associated with tumoral angiogenesis and cell proliferation in a variety of cancer types. Inhibition of TACR 1 was recently described to impede growth of NB cell lines. However, the relevance of TACR1 in clinical settings is unknown. Patients and Methods: We investigated gene expression levels of full-length and truncated TACR1 in 59 neuroblastomas and correlated these data with the patients' clinical parameters such as outcome, metastasis, International Neuroblastoma Staging System (INSS) status, MYCN proto-oncogene, bHLH transcription factor (MYCN) status, gender and age. Results: Our results indicated that TACRI is ubiquitously expressed in neuroblastoma but expression levels are independent of clinical parameters. Conclusion: Our data suggest that TACRI might serve as a potent anticancer target in a large variety of patients with neuroblastoma, independent of tumor biology and clinical stage.
\end{abstract}

Neuroblastoma (NB), an embryonal malignancy arising from the aberrant growth of neural crest progenitor cells of the sympathetic nervous system, is the most common solid extracranial tumor found in infancy and childhood (1-3). NB is characterized by its clinical heterogeneity: whereas very young children often demonstrate spontaneous tumor regression, older children frequently suffer from progressive disease with poor outcome. Although recent therapeutic

Correspondence to: Michael Berger, MD, Ph.D., Department of Pediatric Surgery, Dr. von Hauner Children's Hospital, Lindwurmstr. 4, 80337 München, Germany. Tel: +49 89440052811, Fax: +49 89440054726, e-mail: michael.fabian.berger@gmail.com

Key Words: Neurokinin-1 receptor, TACR1, tachykinin receptor 1, neuroblastoma, cancer, outcome. approaches in multimodal therapies demonstrated better overall survival, more than $50 \%$ of children with high-risk disease do not respond to modern chemotherapy regimens, resulting in progressive disease. Overall, NB accounts for $12 \%$ of all pediatric oncological deaths $(1,3-6)$.

On the molecular level, several mutations have been discovered for NB. For this and other reasons, NB has served as a paradigm for biological risk assessment and treatment assignment. For example, amplification of MYCN protooncogene, bHLH transcription factor $(M Y C N)$ and hemizygous deletion of chromosomes $1 \mathrm{p}$ and $11 \mathrm{q}$ are found in up to $30 \%$ of patients with NB and are known to correlate with worse outcome and poor prognosis(2). However, the exact mechanisms of tumor formation and progression are still incompletely understood and other oncogenic drivers of tumorigenesis are still to be discovered (2).

Substance P (SP)-tachykinin receptor 1 (TACR1) (also named neurokinin-1 receptor) complex, which acts as a neuronal transmitter, has been linked to inflammation and cell migration (7). Furthermore, several studies have reported that activation of TACR1 (through binding of SP) is associated with tumoral angiogenesis and cell proliferation (8). Among the three subtypes of tachykinin receptors, TACR1 has the highest affinity for the ligand SP (9).

Two splice variants of TACRl are known: a full-length variant (fl-TACRl) and a truncated variant (tr-TACRl). The latter lacks 100 amino acids at its C-terminal end, which serves as a substrate for $\mathrm{G}$ protein receptor kinases and has been reported to differ in its biological function in interaction with SP (10). As a consequence of splicing, the truncated variant lacks sensitivity for negative feedback inhibition, leading to constant activation of the receptor complex(11). Several studies revealed the importance of tr-TACR1 in cancer (10-13).

Importantly, the SP-TACR 1 receptor system was recently described to be a potent anticancer target in NB (14). In the present study, we investigated the role and clinical relevance of fl-TACRl and tr-TACRl in tumor samples from children with NB. 


\section{Patients and Methods}

Patients and tumor tissues. Between 2009 and 2014, 59 patients with NB underwent either biopsy or surgical resection in our Department. Patients were all treated according to the protocol of the German Society of Pediatric Oncology and Hematology (GPOH, study protocol NB2004). For this study, the patients' charts were reviewed retrospectively regarding clinical information: age, gender, International Neuroblastoma Staging System (INSS) status, outcome, metastasis, MYCN status, histology, and extent of surgical resection were obtained and correlated with gene-expression data.

Extent of surgical resection was noted as biopsy only when less than $50 \%$ of the tumor mass was removed. An incomplete resection was defined as when more than $50 \%$ but less than $89 \%$ of the tumor was macroscopically removed. Resection of more than $90 \%$ of tumor but still with visible tumor remnants (90-99\%) was considered a near-complete resection. Removal of $100 \%$ macroscopical tumor mass was considered complete resection (15).

This study was approved by the Institutional Ethics Committee of the University Hospital (LMU Munich; N. 431-11). Written consent was given by the patients' parents for collection of data and laboratory analysis.

RNA extraction and reverse transcription. In essence, samples were treated with TRIzol ${ }^{\circledR}$ reagent for isolation of RNA, according to the manufacturer's instructions (Invitrogen Life Technologies, Carlsbad, CA, USA). RNA was dissolved in RNase-free water. Reverse transcription of RNA-samples ( $2 \mu \mathrm{g}$ each) was performed utilizing SuperScriptTM II reverse transcriptase (Invitrogen Life Technologies), as recommended by the supplier.

Reverse transcription polymerase chain reaction (RT-PCR). Two microliters of cDNA sample were utilized in each PCR reaction with the following specific primers for fl-TACRl and tr-TACRl as well as TATA-box-binding-protein $(T B P)$ housekeeping gene: For fl-TACR1: Forward, 5'-AACCCCATCATCTACTGCTGC-3' and reverse, 5'ATTTCCAGCCCCTCATAGTCG-3' (NM_001058.3); for tr-TACR1 forward, 5'-GGGCCACAAGACCATCTACA-3' and reverse, 5'AAGTTAGCTGCAGTCCCCAC-3' (NM_015727.2); and for TBP: forward, 5'-GCCCGAAACGCCGAATAT-3' and reverse, 5'CCGTGGTTCGTGGCTCTCT-3'. Samples for amplification reactions had a final volume of $20 \mu$ l. iTaq SYBR-green Supermix (Bio-Rad Laboratories, Hercules, CA, USA) was used for amplification reactions. Samples were incubated at $95^{\circ} \mathrm{C}$ for $7 \mathrm{~min}$, followed by 40 cycles of $95^{\circ} \mathrm{C}$ for $30 \mathrm{~s}, 60^{\circ} \mathrm{C}$ for $30 \mathrm{~s}$ and $72^{\circ} \mathrm{C}$ for $30 \mathrm{~s}$, with the final extension cycle performed at $72^{\circ} \mathrm{C}$ for $7 \mathrm{~min}$. The transcript numbers were normalized according to the expression of the housekeeping gene. Relative quantification of gene expression was performed using the $2-\Delta \Delta \mathrm{Ct}$ method as described by Pfaffl (16).

Statistical analysis. Results are given as the mean \pm standard error of the mean. Gene-expression levels of tumor samples are displayed as dot plots for each group. Correlation analysis was performed using a Mann-Whitney $U$-test and a standard $t$-test. Differences with a p-value of less than 0.05 were considered as statistically significant. Statistical calculations were performed using biostatics software from GraphPAD Prism ${ }^{\circledR}$ (La Jolla, CA, USA).

Kaplan-Meier curves were used for demonstration of overall survival (OS), and two-sided log-rank test was utilized for comparison of survival curves.
Table I. Patient characteristics.

\begin{tabular}{|c|c|c|}
\hline & Patients (n) & $\%$ \\
\hline \multicolumn{3}{|l|}{ Gender } \\
\hline Female & 26 & 44.1 \\
\hline Male & 33 & 55.9 \\
\hline \multicolumn{3}{|l|}{ Histology } \\
\hline Ganglioneuroma (GN) & 5 & 8.5 \\
\hline Ganglioneuroblastoma (G1) & 19 & 32.2 \\
\hline Differentiated neuroblastoma (G2) & 14 & 23.7 \\
\hline Undifferentiated neuroblastoma (G3) & 21 & 35.6 \\
\hline \multicolumn{3}{|l|}{ Outcome } \\
\hline Alive & 46 & 77.9 \\
\hline Died from disease & 13 & 22.1 \\
\hline \multicolumn{3}{|l|}{ Extent of surgery } \\
\hline Biopsy only & 2 & 3.4 \\
\hline Incomplete resection $(<50 \%-90 \%)$ & 6 & 10.2 \\
\hline Nearly complete resection (>90-99\%) & 24 & 40.7 \\
\hline Complete resection $(100 \%)$ & 27 & 45.7 \\
\hline \multicolumn{3}{|l|}{$M Y C N$ status } \\
\hline Amplified & 6 & 10.2 \\
\hline Not amplified & 53 & 89.8 \\
\hline \multicolumn{3}{|l|}{ Metastasis } \\
\hline Yes & 26 & 44.1 \\
\hline No & 33 & 55.9 \\
\hline \multicolumn{3}{|l|}{ International Neuroblastoma Staging System } \\
\hline 1 & 6 & 10.2 \\
\hline $2 \mathrm{a}$ & 6 & 10.2 \\
\hline $2 b$ & 1 & 1.7 \\
\hline 3 & 20 & 33.9 \\
\hline 4 & 25 & 42.3 \\
\hline $4 \mathrm{~s}$ & 1 & 1.7 \\
\hline
\end{tabular}

MYCN: MYCN proto-oncogene, bHLH transcription factor.

\section{Results}

TACRl shows high expression in human NB. Geneexpression profiles of fl-TACRl and tr-TACRl were investigated in human NB samples. In general, fl-TACRI showed a low expression profile, whereas tr-TACRl demonstrated much higher expression levels in NB samples (Figure 1a). This difference was statistically significant $(p<0.0001)$. However, fl-TACRl and tr-TACRl expression did not demonstrate a significant correlation $(\mathrm{r}=0.2201$, $p=0.0969$ ) (Figure1b). Furthermore, we performed an analysis of the association between expression of each splice variant (with cutoff as the overall mean expression) and overall prognosis. There was no statistically significant difference for either splice variant (Figure 1c).

Clinical characteristics. The mean patient age at diagnosis was 40.5 months, with a range from 0 months to 19.2 years. Twenty-six patients $(44.1 \%)$ had metastases at diagnosis. For further patient characteristics, see Table I. 
a

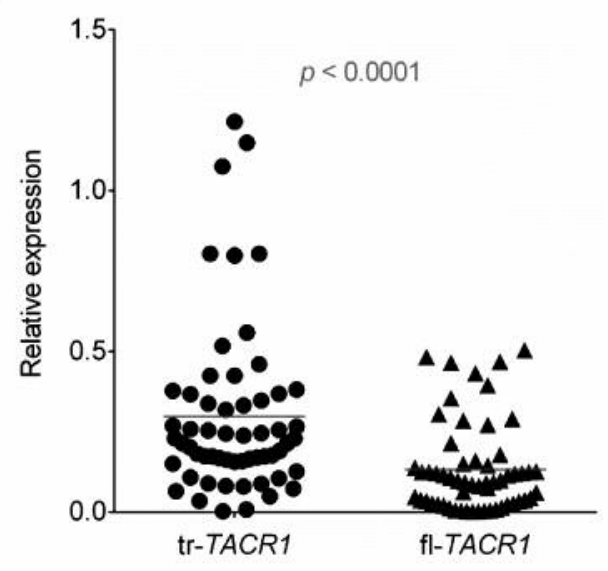

C

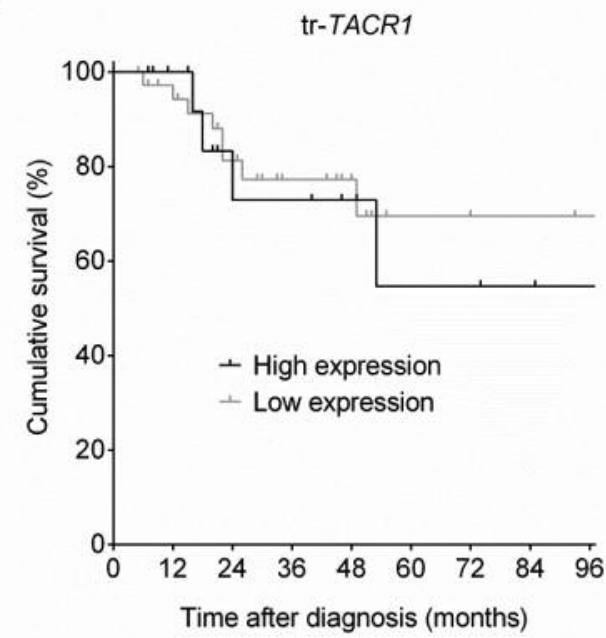

b

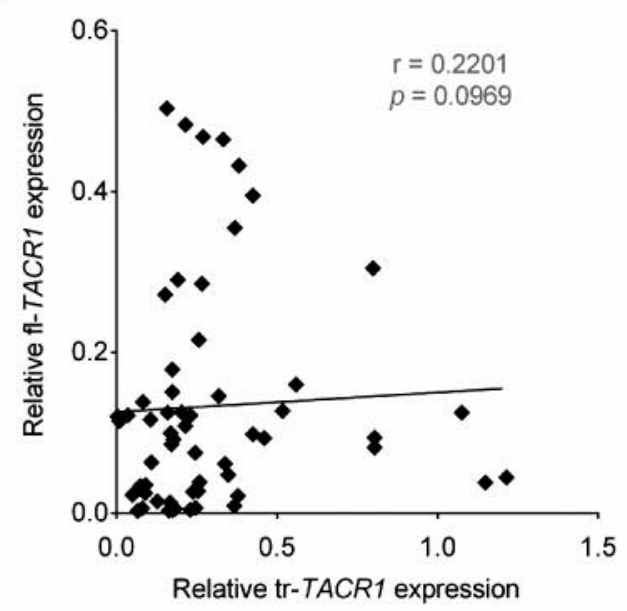

fl-TACR1

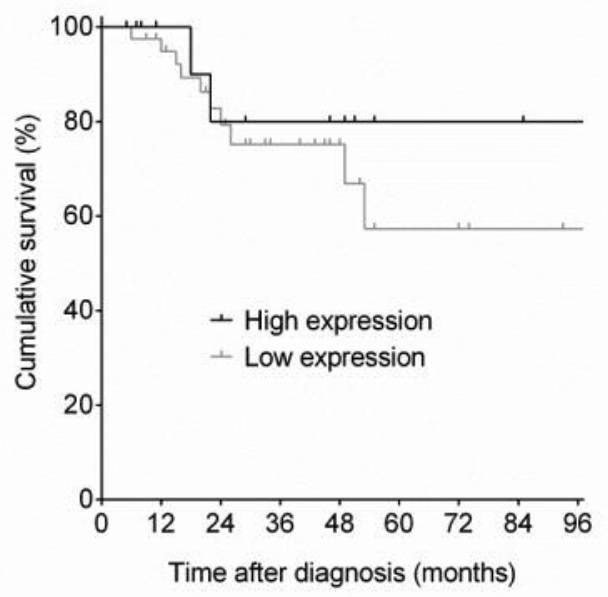

Figure 1. a: Gene-expression of full-length tachykinin receptor 1 (fl)-TACR1 and truncated (tr)-TACR1 in human neuroblastoma samples. b: Correlation between fl-TACR1 and tr-TACR1. c: Association between expression of each splice variant (with cut-off as the overall mean expression) and overall prognosis.

Gene-expression levels of fl-TACR1 and tr-TACR1. Both flTACRl and tr-TACRl variants as well as their ratio were analyzed for association with clinical and biological findings of NB samples. Neither fl-TACRl nor tr-TACRl were significantly associated with histology, age or gender (Figure 2). Clinical parameters such as $M Y C N$ status and metastasis, which are linked with aggressive disease, also demonstrated no significant association with tr-TACRI nor fl-TACRl. Only fl-TACR 1 was significantly higher in INSS stage $4(p=0.0164$, Figure 3), but there was no association for tr-TACR1 nor their ratio. As was the case for the other clinical parameters mentioned, the analysis of gene-expression levels did not show any significant association with outcome (Figure 3).

\section{Discussion}

An abundance of studies have proven the importance of the SP-TACR 1 complex in cancer and disease progression (7, 10). Moreover, during the past decade, TACRI and its high expression in a variety of cancer types has become the focus of attention for targeted therapy. Tachykinin-receptor antagonists such as aprepitant, currently used as a clinical drug for the treatment of chemotherapy-related nausea and vomiting, in several reports demonstrated in vitro and in vivo efficacy against TACR1, resulting in a robust anticancer effect $(12,14,17)$. Similar effects have been observed for other TACR 1 antagonists. 
Truncated
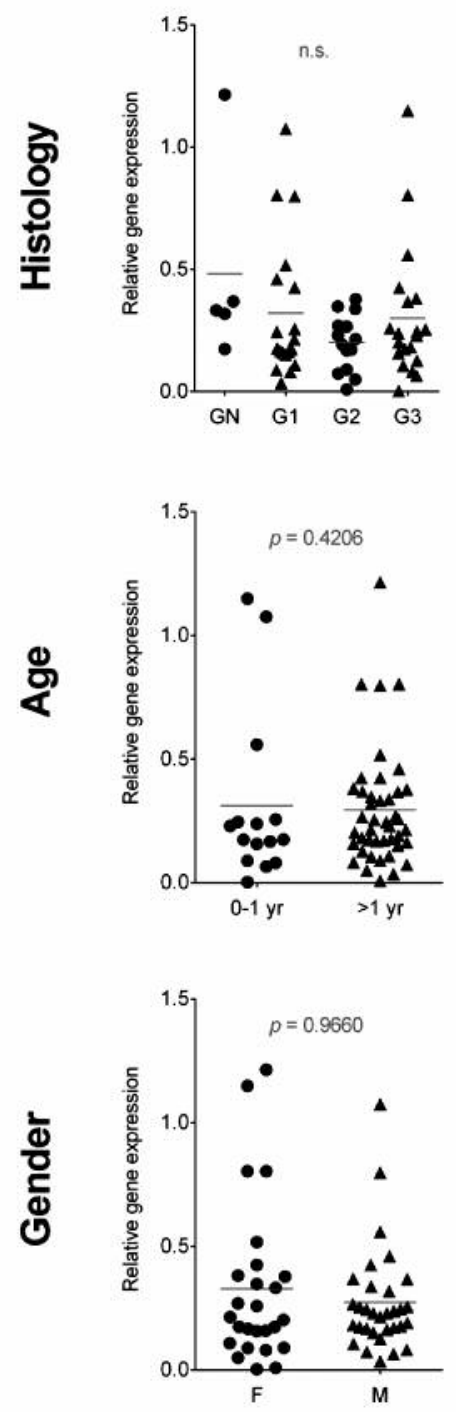

Full-length
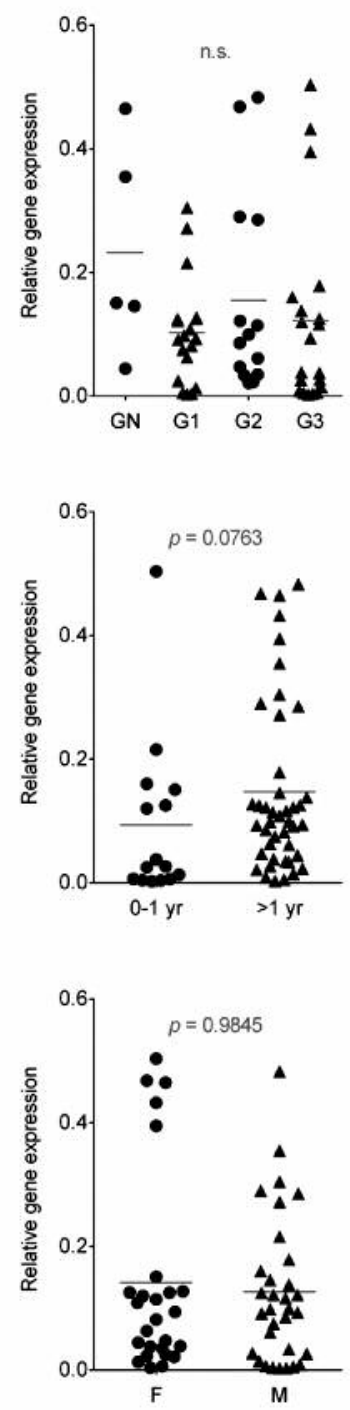

Ratio
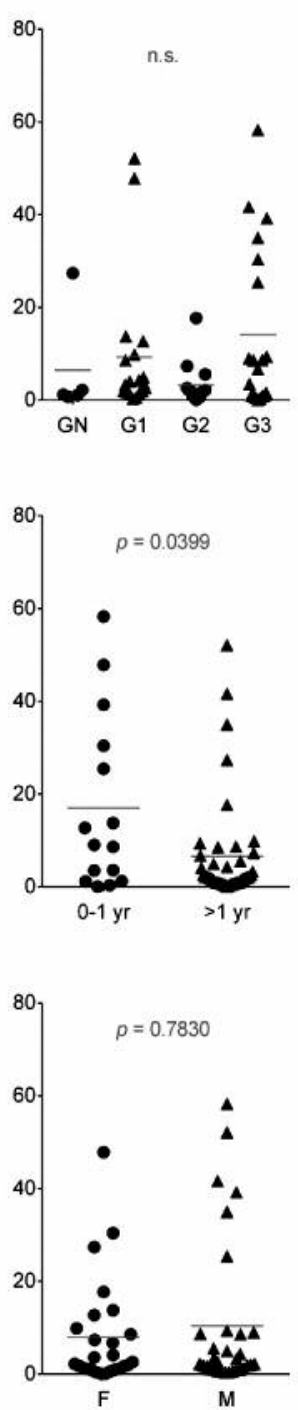

Figure 2. Gene-expression of full-length tachykinin receptor 1 (fl)-TACR1 and truncated (tr)-TACR1, and their ratio in human neuroblastoma samples according to histology, age and gender. GN: Ganglioneuroma, G1: gangioneuroblastoma, G2: differentiated neuroblastoma, G3: undifferentiated neuroblastoma, yr: year, F: female, M: male.

Little is known about the role of TACRl in oncogenesis and tumor progression in NB. Nowicki et al. investigated the cell immunophenotype of metastatic and primary tumors in NB and found, among other nervous tissue markers, high expression of SP in both groups (18). In 2005, Munoz et al. reported TACRI expression in two NB cell lines (19). Perhaps the most sophisticated study regarding this topic so far was recently published by Henssen et al. The authors examined tachykinin receptors in a panel of NB cell lines. Their results suggest TACRl expression in all tested cell lines, even though expression levels varied. Our previous studies in hepatoblastoma are consistent with this finding $(12,14)$. In a second step, growth inhibition was induced with targeting NB cells in vivo and in vitro (mouse xenograft model) with fosaprepitant, a tachykinin receptor antagonist similar to aprepitant. The Authors concluded that gene expression of TACR 1 is increased in NB cell lines resulting in expression of this receptor, making it an attractive target for targeted therapy with TACR1 antagonists (14). Interestingly, NB is currently the only solid cancer in 


\section{Truncated}
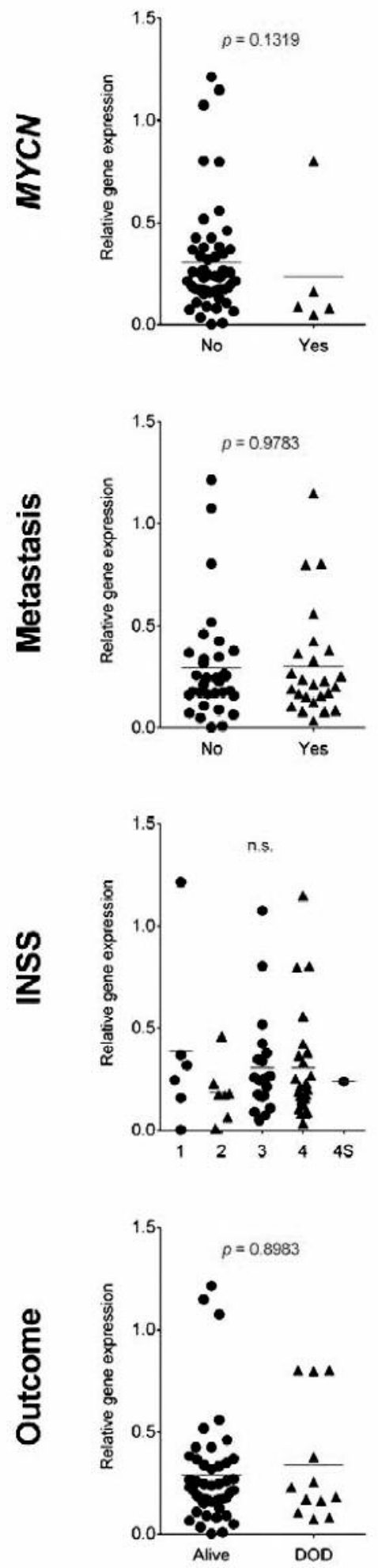

Full-length
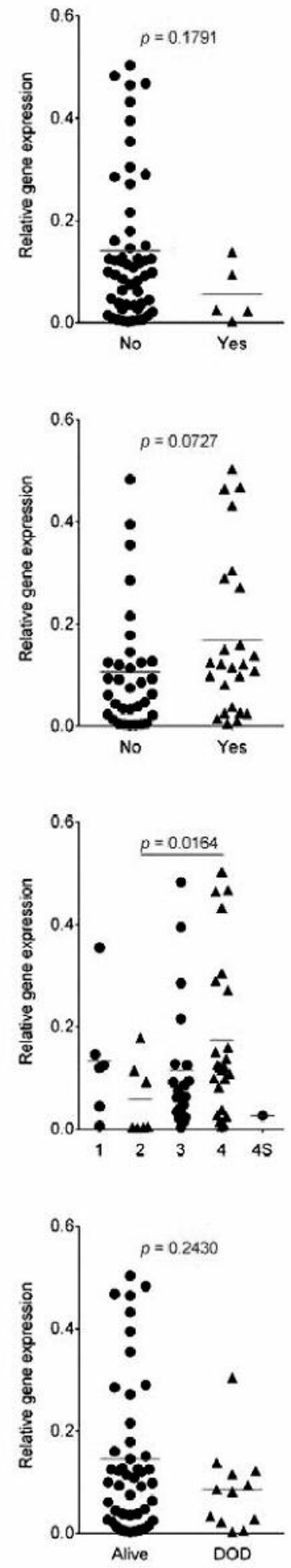

Ratio
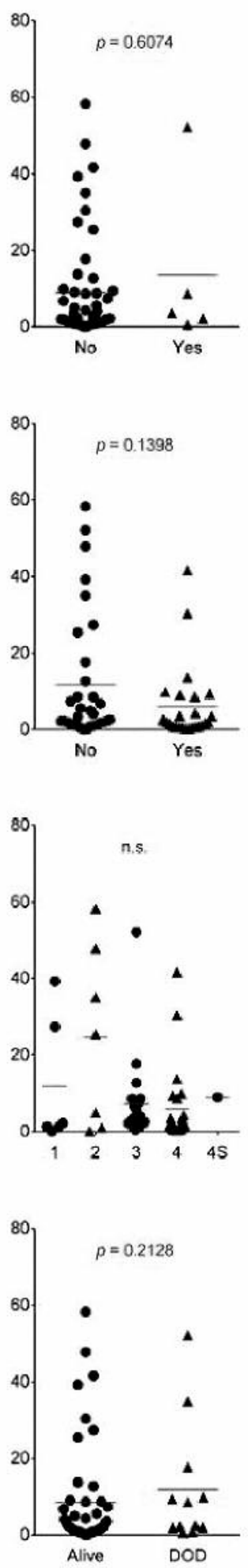

Figure 3. Gene-expression of full-length tachykinin receptor 1 (fl)-TACR1 and truncated (tr)-TACR1, and their ratio in human neuroblastoma samples according to MYCN proto-oncogene, bHLH transcription factor (MYCN) status, presence of metastasis, International Neuroblastoma Staging System (INSS), and outcome. DOD: Died of disease. 
childhood for which a positive therapeutic effect has been proven for targeted therapy (via GD2). This understanding raises hope that there could potentially be other targets via which a similarly promising effect can be achieved.

In the present study, we demonstrated ubiquitous gene expression of fl-TACRl and tr-TACRl in a cohort of children with NB. Our results suggest increased gene expression of trTACRl and low expression of fl-TACRl in NB tumor tissue. Interestingly, gene expression levels were independent of clinical markers such as INSS stage, MYCN status and histology. These findings are in accordance with what has been found for other tumor types. As was briefly mentioned above, it is generally understood that it is the expression of the truncated splice variant that is dominantly involved in cancer formation $(20,21)$. This finding strongly correlates with our own data published recently for the expression profile of the SP-TACR1 receptor system in hepatoblastoma, another aggressive childhood cancer $(11,12)$. Compared to normal liver tissue, we found that gene expression levels of fl-TACRI and tr-TACRl were both increased, whereas the level of trTACR I was significantly increased compared to fl-TACRl ( $p=0.0301$ ) (11). Similar to the results presented here for NB, in hepatoblastoma, we found no correlation between the expression profile of TACRI and clinical behavior or disease stage. Yet TACR1 antagonists had a remarkable therapeutic effect on hepatoblastoma growth both in vitro and in vivo.

Our findings presented in this study are furthermore in accordance with those of other studies. Chen et al. examined expression of SP and TACRl in tissue microarrays of colorectal cancer and adjacent healthy tissue with immunohistochemistry. Expression of SP and TACRI were both significantly increased in samples of patients with lymph node metastasis; furthermore, high TACR 1 expression was also correlated with TNM stage III and IV. Besides these significant correlations, expression of SP and TACRl was not associated with clinical parameters such as age, sex, gender, distant metastasis and pathological grading (7).

Somewhat different results were found in a recent study in breast cancer (10). The different role of fl- and tr-TACRl in breast cancer cell lines and breast cancer tumor samples were investigated. The authors found high gene-expression levels of tr-TACRl in cells of advanced malignancy, whereas flTACR I demonstrated mainly high gene-expression levels in normal breast and mesenchymal cells; in breast cancer cells, gene expression of fl-TACRl was markedly reduced (10). Moreover, results suggest that fl-TACRl was inversely correlated with invasiveness, proliferation and metastasis, whereas high expression of tr-TACRl seemed to affect progressive disease and metastasis. Interestingly, we found similar results when investigating hepatoblastoma (12), however, as mentioned above, we did not find any correlation between disease stage or prognosis for the data presented here in NB nor for hepatoblastoma presented previously.
As all scientific studies, ours has several flaws, potentially biasing our data. Firstly, we only investigated gene-expression levels not protein-expression levels. Just as there are variations in the splice products prior to expression, there could be mRNA abrogation or other mechanisms that alter the expression profile that we did not account for. The solution to this would be immunohistochemical staining of our samples in order to correlate the protein-expression profile with the mRNA profile. Although such staining is generally feasible, it is increasingly challenging given the unavailability of appropriate antibodies against the tr-TACRl (20). Thirdly, NB is a very heterogeneous tumor. By taking one sample per tumor, the examined areas might not be representative of the whole specimen. Furthermore, we lacked a control group with healthy tissue to compare gene-expression levels simply due to the nature of the tumor type we investigated.

Nevertheless, despite these limitations, we feel that our findings support evidence that NB tissue, similarly to other cancer types, ubiquitously expresses TACRl. This understanding could hold tremendous clinical relevance and make TACR1 an attractive therapeutic target for a large variety of clinical and biological NB subsets. Further research is needed to clarify the exact clinical and biological role of TACRl in the oncogenesis of childhood NB.

\section{Acknowledgements}

The Authors are grateful to Fatemeh Promoli for technical assistance.

\section{References}

1 Bosse KR and Maris JM: Advances in the translational genomics of neuroblastoma: From improving risk stratification and revealing novel biology to identifying actionable genomic alterations. Cancer 1221: 20-33, 2016.

2 Pugh TJ, Morozova O, Attiyeh EF, Asgharzadeh S, Wei JS, Auclair D, Carter SL, Cibulskis K, Hanna M, Kiezun A, Kim J, Lawrence MS, Lichenstein L, McKenna A, Pedamallu CS, Ramos AH, Shefler E, Sivachenko A, Sougnez C, Stewart C, Ally A, Birol I, Chiu R, Corbett RD, Hirst M, Jackman SD, Kamoh B, Khodabakshi AH, Krzywinski M, Lo A, Moore RA, Mungall KL, Qian J, Tam A, Thiessen N, Zhao Y, Cole KA, Diamond M, Diskin SJ, Mosse YP, Wood AC, Ji L, Sposto R, Badgett T, London WB, Moyer Y, Gastier-Foster JM, Smith MA, Guidry Auvil JM, Gerhard DS, Hogarty MD, Jones SJ, Lander ES, Gabriel SB, Getz G, Seeger RC, Khan J, Marra MA, Meyerson $\mathrm{M}$ and Maris JM: The genetic landscape of high-risk neuroblastoma. Nat Genet 453: 279-284, 2013.

3 Maris JM: Recent advances in neuroblastoma. New Engl J Med 36223: 2202-2211, 2010.

4 Pohl A, Erichsen M, Stehr M, Hubertus J, Bergmann F, Kammer B and von Schweinitz D: Image-defined risk factors correlate with surgical radicality and local recurrence in patients with neuroblastoma. Klin Padiatr 2283: 118-123, 2016. 
5 Oeffinger KC, Mertens AC, Sklar CA, Kawashima T, Hudson MM, Meadows AT, Friedman DL, Marina N, Hobbie W, KadanLottick NS, Schwartz CL, Leisenring W, Robison LL and Childhood Cancer Survivor S: Chronic health conditions in adult survivors of childhood cancer. N Engl J Med 35515: 1572-1582, 2006.

6 Yu AL, Gilman AL, Ozkaynak MF, London WB, Kreissman SG, Chen HX, Smith M, Anderson B, Villablanca JG, Matthay KK, Shimada H, Grupp SA, Seeger R, Reynolds CP, Buxton A, Reisfeld RA, Gillies SD, Cohn SL, Maris JM, Sondel PM and Children's Oncology G: Anti-gd2 antibody with gm-csf, interleukin-2, and isotretinoin for neuroblastoma. N Engl J Med 36314: 1324-1334, 2010.

7 Chen XY, Ru GQ, Ma YY, Xie J, Chen WY, Wang HJ, Wang SB, Li L, Jin KT, He XL and Mou XZ: High expression of substance $\mathrm{p}$ and its receptor neurokinin-1 receptor in colorectal cancer is associated with tumor progression and prognosis. OncoTargets Ther 9: 3595-3602, 2016.

8 Ortiz-Prieto A, Bernabeu-Wittel J, Zulueta-Dorado T, LorenteLavirgen AI and Munoz M: Immunolocalization of substance P and NK-1 receptor in vascular anomalies. Arch Dermatol Res 3092: 97-102, 2017.

9 Douglas SD and Leeman SE: Neurokinin-1 receptor: Functional significance in the immune system in reference to selected infections and inflammation. Ann NY Acad Sci 1217: 83-95, 2011.

10 Zhou Y, Zhao L, Xiong T, Chen X, Zhang Y, Yu M, Yang J and Yao Z: Roles of full-length and truncated neurokinin-1 receptors on tumor progression and distant metastasis in human breast cancer. Breast Cancer Res Treat 1401: 49-61, 2013.

11 Garnier A, Ilmer M, Becker K, Haberle B, D VONS, Kappler R and Berger M: Truncated neurokinin-1 receptor is an ubiquitous antitumor target in hepatoblastoma, and its expression is independent of tumor biology and stage. Oncol Lett 111: 870$878,2016$.

12 Berger M, Neth O, Ilmer M, Garnier A, Salinas-Martin MV, de Agustin Asencio JC, von Schweinitz D, Kappler R and Munoz M: Hepatoblastoma cells express truncated neurokinin-1 receptor and can be growth inhibited by aprepitant in vitro and in vivo. $\mathrm{J}$ Hepatol 605: 985-994, 2014.

13 Cordier D, Gerber A, Kluba C, Bauman A, Hutter G, Mindt TL and Mariani L: Expression of different neurokinin-1 receptor (NK1R) isoforms in glioblastoma multiforme: Potential implications for targeted therapy. Cancer Biother Radiopharm 295: 221-226, 2014.
14 Henssen AG, Odersky A, Szymansky A, Seiler M, Althoff K, Beckers A, Speleman F, Schafers S, De Preter K, Astrahanseff K, Struck J, Schramm A, Eggert A, Bergmann A and Schulte JH: Targeting tachykinin receptors in neuroblastoma. Oncotarget 81 : 430-443, 2017.

15 Simon T, Haberle B, Hero B, von Schweinitz D and Berthold F: Role of surgery in the treatment of patients with stage 4 neuroblastoma age 18 months or older at diagnosis. J Clin Oncol 316: 752-758, 2013.

16 Pfaffl MW: A new mathematical model for relative quantification in real-time rt-pcr. Nucleic Acids Res 299: e45, 2001.

17 Bigioni M, Benzo A, Irrissuto C, Maggi CA and Goso C: Role of NK-1 and NK-2 tachykinin receptor antagonism on the growth of human breast carcinoma cell line MDA-MB-231. Anticancer Drugs 1610: 1083-1089, 2005.

18 Nowicki $M$ and Miskowiak B: Comparison of the cell immunophenotype of metastatic and primary foci in stage IV-S neuroblastoma. Folia Histochem Cytobiol 403: 297-303, 2002.

19 Munoz M, Rosso M, Perez A, Covenas R, Rosso R, Zamarriego $\mathrm{C}$ and Piruat JI: The nk1 receptor is involved in the antitumoural action of 1-733,060 and in the mitogenic action of substance $P$ on neuroblastoma and glioma cell lines. Neuropeptides 394: 427-432, 2005.

20 Gillespie E, Leeman SE, Watts LA, Coukos JA, O’Brien MJ, Cerda SR, Farraye FA, Stucchi AF and Becker JM: Truncated neurokinin-1 receptor is increased in colonic epithelial cells from patients with colitis-associated cancer. Proc Natl Acad Sci USA 10842: 17420-17425, 2011.

21 Patel HJ, Ramkissoon SH, Patel PS and Rameshwar P: Transformation of breast cells by truncated neurokinin-1 receptor is secondary to activation by preprotachykinin-a peptides. Proc Natl Acad Sci USA 10248: 17436-17441, 2005.
Received August 6, 2017

Revised September 15, 2017

Accepted September 19, 2017 